DOI: 10.20472/IAC.2018.044.023

\title{
TOMOKI KOBAYASHI
}

Aoyama Gakuin University, Japan

TAKASHI KABURAGI

Aoyama Gakuin University, Japan

SATOSHI KUMAGAI

Aoyama Gakuin University, Japan

TOSHIYUKI MATSUMOTO

Aoyama Gakuin University, Japan

YOSUKE KURIHARA

Aoyama Gakuin University, Japan

\section{ANXIETY LEVEL ESTIMATION FROM WEIGHTED FRONTAL LOBE AREA LATERALITY INDEX AT REST USING NIRS}

\begin{abstract}
:
Recently, the number of persons who suffer from mental illnesses that can be caused by excessive stress, such as depression and schizophrenia, has been increasing year by year. To detect and treat these illnesses earlier, it is important to assess the anxiety state of the patients as accurately as possible. In general, the State-Trait Anxiety Inventory (STAI) score is utilized to determine the anxiety state. However, since the STAI is a subjective questionnaire, some patients would intentionally not provide honest answers to the questions. Hence, it is difficult to grasp the patients' anxiety state accurately. To address this, some studies have proposed an estimation method for the STAI score by utilizing the cerebral blood volume, which cannot be intentionally controlled by the patient. In previous studies, the STAI score was estimated from the cerebral blood volume measured in a small area of the prefrontal lobe. To improve the estimation accuracy, we have proposed an estimation method based on the cerebral blood volume measured in a larger area of the prefrontal lobe.

In this study, we not only utilize the cerebral blood volume measured in a larger area, but also aim to improve the estimation accuracy of the STAI score by applying a weight to each area of the prefrontal lobe. We introduce the weighted-multi-channel Laterality Index at Rest (wmc-LIR), which is calculated based on the oxyhemoglobin concentrations measured by near-infrared spectroscopy (NIRS) with $\mathrm{n}$ channels. These are located at measurement points in the prefrontal lobe defined by the international 10-20 system. The weight for each area represents the activity level of the brain in that area.

To confirm the applicability of the proposed method, we validated wmc-LIR using NIRS recordings of 24 subjects. The average absolute error of the proposed method was 4.01. In contrast, the average absolute error of the method proposed in the previous study without assigning weights to brain areas in the same setting was 5.08 .
\end{abstract}

\section{Keywords:}

State-Trait Anxiety Inventory (STAI), cerebral blood volume, near-infrared spectroscopy (NIRS), anxiety state, Liberality Index at Rest (LIR) 
JEL Classification: 110 